\title{
REVIEWS
}

Maciej KoźLik ${ }^{1, \text { A-D, F, Piotr WóJCicki }}{ }^{1,2, \text { A, C, E, F }}$

\section{The Use of Stem Cells in Plastic and Reconstructive Surgery ${ }^{\star}$}

${ }^{1}$ Department of Plastic Surgery, Wroclaw Medical University, Polanica-Zdrój, Poland

${ }^{2}$ Department of Plastic Surgery, Specialist Medical Center, Polanica-Zdrój, Poland

A - research concept and design; B - collection and/or assembly of data; C - data analysis and interpretation;

$\mathbf{D}$ - writing the article; $\mathbf{E}$ - critical revision of the article; $\mathbf{F}$ - final approval of article; $\mathbf{G}$ - other

\begin{abstract}
As part of their clinical practice, plastic surgeons perform procedures in the fields of skin and adipose tissue grafting, the reconstruction of compound tissue loss and congenital malformations, the treatment of acute and chronic wounds and burns, as well as cosmetic surgery. On account of the great expectations associated with their use in therapy, stem cells (SCs) are increasingly frequently employed in in vitro experiments, animal models and clinical trials. The most commonly utilized SCs for these purposes are adult stem cells (AS), which are present in small amounts in every tissue; among these are two types of cells: bone marrow mesenchymal stem cells (BM-MSCs) and adipose-derived stem cells (ADSCs). The aim of this review is to present current findings in experimental research on the use of stem cells in the field of plastic and reconstructive surgery. Many studies have described the progress of trials using ADSCs and BM-MSCs mainly in the treatment of compound tissue loss and chronic wounds. The use of their paracrine, proangiogenic and osteogenic functions is emphasized. However, because of the very high harvesting and culturing expenses and the limited availability of data on their safety for human use, SCs are not a first-choice therapy. Further developments in SC research and gradually decreasing costs mean that along with commercial-scale SC culturing, the use of SCs will become a viable alternative to traditional surgical procedures (Adv Clin Exp Med 2014, 23, 6, 1011-1017).
\end{abstract}

Key words: stem cells, adult stem cells, plastic surgery.

Stem cells (SCs) are a population of undifferentiated cells characterized by the ability to undergo self-renewal and differentiation into various types of tissue. The aim of this review is to present current findings in experimental research on the use of stem cells in the field of plastic and reconstructive surgery.

\section{Types of Stem Cells}

On the basis of their differentiation potential, stem cells are classified as totipotent (or omnipotent), pluripotent, multipotent and unipotent. Omnipotent SCs are present at the earliest stages of ontogenesis and can transform into all forms of embryo and placenta tissue.
Pluripotent cells can be harvested from the inner layers of blastocysts; they produce cells that arise from all 3 germ layers (ectoderm, endoderm and mesoderm). Multipotent SCs can be found in almost all tissue. For a long time it was believed that they could only transform into the cells of one germ layer (e.g., that only hepatic SCs could transform into hepatocytes or bile ducts), but further studies have revealed that some multipotent cells have the same potential as pluripotent SCs. Unipotent stem cells have the lowest potential, and generate only one cell type (e.g., epidermal stem cells only produce terminal keratinized squamous epithelial cell types) [1].

Stem cells can also be classified into 4 groups corresponding to their different origins: embryonic stem cells (ESCs), fetal stem cells, adult stem cells (AS) and induced pluripotent stem cells (iPSCs).

\footnotetext{
* The project is co-financed by the European Union, as part of the European Social Fund.
} 
The zygote - the result of fertilization - is characterized by totipotency. After 4 days of growth, it transforms into a blastocyst. The inner layer of the blastocyst is made of ESCs, which are pluripotent. ESCs can proliferate almost without limit, and are identified by specific transcription factors: NANOG and Oct4 [2]. With the use of specific media and growth factors, ESCs have been cultured in vitro into other cell types, including skeletal muscle, endothelial cells, chondrocytes and myocardiocytes, among others $[3,4]$. In later experiments on these stem cells, researchers have produced eyeball and vocal cord structures $[5,6]$.

In view of the ethical and social dilemmas involved, collecting, culturing and experimenting on embryonic and fetus SCs is legally restricted in many countries [7]. Moreover, some experiments with ESCs have led to cancerous cells and teratoma formation. This is probably related to imperfect methods and current technological limitations, which mean that it is as yet impossible to isolate pure lines of human ESCs [8].

For these reasons, adult stem cells appear to be the simplest to obtain, and have the greatest importance for medical purposes. AS are specific to each organ, and are present in every tissue in small amounts. For example, hematopoietic stem cells (HSC) make up about $0.01-0.001 \%$ of blood cells [9]. Morphology and protein markers allow each AS type to be classified as the tissue it originates from. AS were previously supposed to be multipotent, but are now known to have the potential of ESCs, that is, pluripotency. Due to the phenomenon known as the "plasticity" or "transdifferentiation" of AS, some of these cells can produce cells of a new type when moved to other tissues. Studies by Yamanaka et al. showed that even mature somatic cells, when exposed to specific pluripotency-associated genes, can "regain" the pluripotency of ESCs and differentiate into cells of any germ layer. These experiments were first carried out on mouse fibroblasts, and later on human fibroblasts [10]. For this discovery, Yamanaka was awarded the Nobel Prize in Physiology or Medicine in 2012. The cells have been called induced pluripotent stem cells.

The latest discovery in the field of SCs involves cells that display stimulus-triggered acquisition of pluripotency, which are called STAP cells. Researchers were able to reprogram somatic cells into pluripotent cells by exposing them to sublethal stimuli (low pH). Moreover, STAP cells can develop into both embryonic and placental tissues [11]. These discoveries may perhaps help in exploiting the full potential of ESCs without the associated problems.

Of all SCs, multipotent mesenchymal stem cells (MSCs) have the widest application in clinical practice. From among mesenchymal cells, bone marrow mesenchymal stem cells (BM-MSCs) were obtained earliest and remain in common use. However, obtaining them involves an invasive and inefficient procedure. New procedures have been developed to harvest various types of MSCs from other structures of the human body, including from adipose tissue (adipose-derived stem cells, ADSCs), umbilical cord blood (UCB), periostium, tendons, muscles (muscle-derived stem cells, MDSCs), mucous membranes and skin [9]. Comparative analyses of all types of MSCs do not reveal any major differences between their abilities to differentiate into particular tissues [12].

Apart from their differentiation potential, mesenchymal SCs also possess paracrine signaling functions. They support tissue neovascularization by releasing growth factors, such as vascular endothelial growth factor (VEGF), transforming growth factor (TGF) and hepatocyte growth factor (HGF), into the nearby environment. In experiments on myocardial tissues following cardiac infarction, they have repaired scarred myocardium, increased angiogenesis and, as a result, preserved the entire heart muscle function [13].

The most commonly used SCs are obtained from adipose tissue, on account of the easy and frequently employed acquisition procedure (through liposuction or lipectomy), the low donor-site morbidity rate and the effectiveness of the method. It has been noted that $300 \mathrm{~mL}$ of harvested adipose tissue gives $2-3 \times 10^{8} \mathrm{ADSCs}$, which is from 100 to 1000 times greater than the yield of BM-MSCs that could be acquired from the same volume of bone marrow [14]. The separation of lipoaspirate and ADSC isolation is accomplished by enzymatic collagenase digestion with later centrifugation. Clean lines of ADSCs can be cultured prior to transmitting them to the body.

\section{The Use of Selected SC Fractions in Plastic and Reconstructive Surgery}

Autogenous fat transplants, whose resorption rate exceeds $70 \%$, are traditionally used for the correction of facial symmetry and as a filler for the loss of soft tissues, including correction of atrophy, Parry-Romberg syndrome, distortions after cancer treatment, and the consequences of radiotherapy. Other products that can be applied for these purposes - such as xenogeneic collagen, biopolymers and acellular dermal matrix (ADM) - can induce immunological reactions at the recipient site. The characteristics of the ideal filler for soft tissues are 
that it should be easy to use, effective and low-cost, and that it should provide long-lasting effects without inducing any immunological reaction.

In order to decrease the percentage of fat transplant resorption, techniques for enriching transplants with stem cells are employed. The staged cell-enriched tissue injection (SET) technique consists of 2 phases: first, the patient receives a conventional adipose tissue transplant. Second, part of the lipoaspirate is prepared to obtain ADSCs, which can be mixed with platelet-rich plasma (PRP). This solution is later injected into the sites filled with the fat transplant. Tiryaki et al. [15] described the application of SET in 29 patients, including postmastectomy patients ready for breast reconstruction, women who qualified for breast augmentation, and patients with Parry-Romberg syndrome, mutilation after poliomyelitis, and facial scars. After 8 weeks of injections, atrophy of the injected tissue was minimal; the only complication involved bruises in a few cases that healed after 2-3 weeks. The addition of ADSCs to fat transplant has been demonstrated to act in a proangiogenic and antiapoptotic manner and to reduce local inflammation [16]. However, it is still unclear whether the use of stem cells is safe for patients who have undergone oncological operations. There are justified concerns that injecting SCs into the operative site may lead to an acceleration of tumorigenesis. In some in vitro experiments, ADSCs increased the invasiveness of breast cancer cells [17].

Simultaneous transplantation of fat with stem cells harvested from muscles has proved to be beneficial. Experiments in animal models show that MDSCs also cause increases in the number of blood vessels and reductions in fat tissue resorption [18]. Initial data also suggests that the addition of ADSCs significantly improves the viability of cartilage grafts [19].

Stem cells can be used to fill bone cavities. In a few in vitro experiments, bone substitutes were combined with BM-MSCs and endothelial progenitor cells (EPCs). It was shown that MSCs can attach to artificial scaffolds that prolong their vitality [20]. Furthermore, a few in vivo studies have indicated that ADSCs secrete large amounts of bone morphogenetic proteins (BMPs), which accelerate osteogenesis [21]. However, a study by Stockmann et al. [22] failed to reveal no statistical difference in bone reconstruction with SCs compared to autogenous bone. BM-MSCs have also been used in bone distractions in animal models [23]. Injections of stem cells into an artificial fissure in a rat's mandible led to amplified formation of new bone. There have also been a few clinical trials on alveolar cleft reconstruction with bone substitutes and BM-MSCs [24], and also an animal cranioplasty model with adipose-derived stem cells [25].

Human acellular tissue can be used in clinical practice to fill and reconstruct the abdominal wall [26]. However, like fat tissue, it undergoes a significant resorption rate. In order to reduce this complication, experimental animal trials have been carried out in which an acellular dermal matrix was covered with a layer of ADSCs. The results indicate that the addition of stem cells decreases the tissue atrophy level and contributes to the preservation of the graft in long-term follow-ups [27].

Skin flaps with a microvessel pedicle are commonly used in reconstructive techniques. In many situations, they are exposed to prolonged ischemia and can develop an ischemia-reperfusion injury that may lead to flap tissue loss. In one study on rats, ischemia-reperfusion injury conditions were recreated by elevating an extended inferior epigastric artery skin flap. In the experimental groups, the vessel pedicle was clenched for $3 \mathrm{~h}$, and in one group of animals the vessels were injected with ADSCs. It was found that stem cell treatment significantly enhances skin flap survival in the aftermath of ischemia, to an extent that almost equals surgical results without ischemia. This effect is accompanied by a pronounced and significant angiogenic response and improved blood perfusion [28]. If these results can be confirmed by other studies, they will mark substantial progress in microsurgical reconstruction surgery.

The reconstruction of compound anatomical structures that include soft tissue with bone fragments is a great challenge for modern plastic surgery. There are good reasons to believe that, in this particular field of plastic surgery, stem cells may also lead to major breakthroughs. Researchers from Finland have reported a maxilla reconstruction with bone created in vitro using ADSCs [29]. A 65-year-old male who had undergone hemimaxillectomy due to a large recurrent keratocyst underwent liposuction for fat tissue collection. The graft was purified to obtain ADSCs, which were later cultured on a bone substitute (beta tricalcium phosphate, betaTCP) with growth factors. A titanium cage filled with ADSCs and betaTCP was inserted into the patient's left rectus abdominis muscle. After 8 months, the cage was removed and the rectus abdominis free flap with bone neotissue was placed in the maxillary defect. By the 12-month follow-up, the graft had become stable and could serve as the basis for dental implants. In a similar way, surgeons from Germany recreated a 7-centimeter long mandible fragment. In this case, a 56-year-old patient had a titanium mesh cage filled with bone substitute and BM-MSCs inserted into his latissimus dorsi muscle. In the second step, 
a free flap was transplanted to the defect site [30]. There is also a case report of a one-step mandible reconstruction with an adipose stem cell tissueengineered construct. Here, a patient with a recurrent ameloblastoma of the parasymphyseal region of the mandible underwent an extensive tumor resection (the full length of the resected bone fragment was $10 \mathrm{~cm}$ ) and simultaneous reconstruction with a prepared graft containing ADSCs and bone substitute. The transplant was secured with titanium mesh and plates. After 10 months, the new bone was suitable for dental implants [31]. This protocol has been called in situ bone formation. At the 3-year follow-up, there was no sign of bone resorption. If other trials with such complex reconstructive techniques are as successful, they will be applied in the future on a larger scale. However, because of the high costs, only isolated cases have as yet been reported using these techniques.

\section{Treatment of Chronic Wounds and Burns}

The skin is the largest organ of the human body. It serves as a barrier that protects our internal environment from pathogens and plays a major role in thermoregulation. Deep non-healing wounds that involve the full thickness of the skin and deeper structures (such as chronic wounds and burns) are among the greatest challenges to medicine. The incidence of nonhealing cutaneous wounds in the United States alone is 5 to 7 million cases per year, with an annual cost of over 20 billion dollars [32]. The effectiveness of treatment is estimated at $50 \%$.

An experiment with genetically modified mice and BM-MSCs was carried out with the aim of assessing the usefulness of stem cells in diabetic wound treatment [33]. Diabetic leptin receptor-deficient $(\mathrm{db} / \mathrm{db})$ mice employed showed pathologies characteristic of diabetes, such as obesity, hyperglycemia and hyperlipidemia. Full skin thickness $(6 \mathrm{~mm})$ cuts were made on the backs of some of the animals and cultured BM-MSCs were injected into the site. The animals were examined after 7, 14 and 28 days. In histological examinations, the wounds treated with BM-MSCs showed accelerated wound healing, enhanced re-epithelialization and angiogenesis. In addition to transforming into keratinocytes, the stem cells also exerted paracrine effects. Western blot and PCR analysis showed high amounts of the proangiogenic factors VEGF- $\alpha$ and Ang- 1 .

Another technique using SCs is to cover the surface of a wound with a suspension of cells using a fibrin spray. In vitro experiments were carried out by Falanga et al. on animal models (healthy mice and $\mathrm{db} / \mathrm{db}$ mice), and also in clinical trials [34]. The researchers first established the appropriate proportions of fibrinogen, thrombin and other components for the fibrin spray in animal models. They then began clinical trials on patients with acute wounds remaining after the removal of skin cancers (basal cell and squamous cell carcinomas) and with chronic wounds due to venous insufficiency and diabetic neuropathy. For acute wounds, further studies are needed to show the full potential of therapy with BM-MSCs. The results for chronic wounds revealed statistically significant correlations, indicating that the greater the number of applied mesenchymal cells, the larger the reduction in the ulcer. Only applications of more than $1 \times 10^{6} \mathrm{cells} / \mathrm{cm}^{2}$ to the wound were associated with a subsequent decrease (within 2-4 weeks) in ulcer size. These results were also confirmed in animal trials in both the normal and diabetic mice groups. Full-thickness wounds on these animals healed faster when treated with autologous BM-MSCs, as compared to fibrin sprays without any cell component. In the future, stem cell suspensions may possibly be an alternative to the standard treatment of nonhealing ulcers.

Artificial dermal substitutes have been shown to have great therapeutic potential for extensive scars, birthmarks and burns, both in humans and in animal models. The breakthrough work of Japanese doctors has demonstrated that the addition of SCs to collagen sponge may be therapeutically effective and lead to wound closure [35]. Their study involved 20 patients with extensive wounds of various etiologies: burns, deep injury wounds, infections and decubitus ulcers. The patients' ages ranged from 22 to 91 . Prior to the main research, most patients had undergone unresponsive treatment with artificial skin grafting. The wounds of 16 patients were positive for pathogenic bacteria. All the patients were treated with stem cells from bone marrow aspirate with collagen sponge. Soon after the application of the marrow mesenchymal cell/artificial dermis composite graft, the macroscopic and histological results both showed that formation of both cutaneous and subcutaneous tissues had occurred. The wounds healed, completely or partially, in 18 out of 20 patients; in some cases additional skin grafts were necessary. The therapeutic effects of the BM-MSC/artificial dermis composite graft were demonstrated in all the patients. The authors suggested that subcutaneous fibrous tissue and vessels regenerated thanks to the MSCs, since the effect was not seen in patients treated with the artificial dermis alone. The researchers also suggested that a proper scaffold is essential for tissue regeneration. The artificial dermis appears to be an effective scaffolding material. 
This method is minimally invasive, and with a reduction of the expenses associated with the culturing procedure, it could be carried out on a commercial scale.

Other animal-model experiments on artificial skin matrices with the addition of MSCs also support the idea that this combination may be useful in full skin thickness injuries and burn treatment, as it improves vascular density and collagen synthesis [36].

Thermal trauma in a deep-burn injury causes 3 concentric zones of skin damage: the central, irreversibly affected zone of coagulation; the zone of stasis; and the outermost zone of hyperemia. An experimental study on rats demonstrates that subcutaneous injections of allogeneic BM-MSCs into the stasis zone decrease apoptosis count and are beneficial for the survival of the zone. The disadvantage of this method is the necessity of administering the stem cells very soon after the injury. Further research should be performed to demonstrate the clinical efficiency of this treatment [37].

\section{Cosmetic Surgery}

Esthetic surgery is a significant branch of plastic surgery. Many people are willing to undergo surgical operations in order to improve their appearance. Yoshimura et al. showed that ADSCs may be useful in cosmetic breast augmentation, and can also be used as filler after the removal of breast implants [38]. Transplantation of fat tissue has been widely used for this purpose, and the researchers established their own protocol for enriching the aspirate with ADSCs: half of the material from the liposuction was centrifuged to obtain a stem-cell fraction, which was later added to the remainder of the fat tissue. This protocol has been called cell-assisted lipotransfer (CAL). The difference between CAL and the SET technique mentioned above is that the former involves the simultaneous combination of ADSC-poor fat with the stem cell fraction prior to injection into the patient's body. This method provides a lower graft absorption rate in the first 2 months after the operation, with the breast volume showing minimal changes thereafter. Compared with breast augmentation using implants of the same size, CAL augmentation gave less height but a more natural contour to the breasts. The formation of cysts
( $<12 \mathrm{~mm}$ ) was found by magnetic resonance imaging in 2 out of 40 patients, and microcalcification was detected in 2 patients at 24 months. The maximum volume of augmentation was 100 to $200 \mathrm{~mL}$ of enhanced fat tissue, and for that reason, this method is not suitable for large breast augmentation. In another trial, the same authors used the CAL technique on 15 patients who underwent breast implant removal. CAL was applied immediately during a single surgical procedure. The clinical results were satisfactory, and the authors suggest that CAL is a suitable method for replacing artificial breast implants with autologous tissue.

In a more recent report, CAL was used for soft-tissue augmentation (facial recontouring) in patients with craniofacial microsomia. A study of 14 patients showed that this method may be safe and effective, and even superior in clinical application to standard lipoinjection. The surviving fat volume at 6 months was $88 \%$ for the experimental group (CAL) and 54\% for the control group (conventional lipoinjection) [39].

Another trial of the esthetic uses of SCs was presented in a case report on the application of ADSCs in hair-restoration surgery. Unfortunately, tests on 5 patients did not lead to satisfying results. At the 10-month follow-up, no evidence was seen of hair growth in the recipient area [40].

The limitations of stem cell use in cosmetic surgery appear to be caused by the experimental nature of the research and the high costs of acquiring and culturing MSCs, which render them uneconomical for use on a commercial scale.

\section{Conclusions}

This review describes possible applications of multipotent stem cells based on experimental animal tests and isolated clinical trials. The literature currently available supports the conclusion that these techniques are not yet ready for plastic surgeons' general clinical practice. There is still insufficient data on the safety of SCs for human use. At present, short follow-up periods and very high harvesting and culturing expenses disqualify stem cells as a first-choice therapy. However, the rapid development of MSC research will certainly enrich plastic surgeons' operating techniques with new, revolutionary means of treatment.

\section{References}

[1] Beck B, Blanpain C: Mechanisms regulating epidermal stem cells. EMBO J 2012, 31, 2067-2075.

[2] Wang Z, Oron E, Nelson B, Razis S, Ivanova N: Distinct lineage specification roles for NANOG, OCT4, and SOX2 in human embryonic stem cells. Cell Stem Cell 2012, 10, 440-454. 
[3] Rohwedel J, Maltsev V, Bober E: Muscle cell differentiation of embryonic stem cells reflects myogenesis in vivo: developmentally regulated expression of myogenic determination genes and functional expression of ionic currents. Dev Biol 1994, 164, 87.

[4] Maltsev VA, Rohwedel J, Hescheler J, Wobus AM: Embryonic stem cells differentiate in vitro into cardiomyocytes representing sinusnodal, atrial and ventricular cell types. Mech Dev 1993, 44, 41.

[5] Zhu J, Zhang K, Sun Y, Gao X, Li Y, Chen Z, Wu X: Reconstruction of functional ocular surface by acellular porcine cornea matrix scaffold and limbal stem cells derived from human embryonic stem cells. Tissue Eng Part A $2013,19,2412-2425$.

[6] Leydon C, Selekman JA, Palecek S, Thibeault SL: Human embryonic stem cell-derived epithelial cells in a novel in vitro model of vocal mucosa. Tissue Eng Part A 2013, 19, 2233-2241.

[7] Zarzeczny A, Caulfield T: Emerging ethical, legal and social issues associated with stem cell research and the current role of the moral status of the embryo. Stem Cell Rev 2009, 5, 96-101.

[8] Wakitani S, Takaoka K, Hattori T, Miyazawa N, Iwanaga T, Takeda S, Watanabe TK, Tanigami A: Embryonic stem cells injected into the mouse knee joint form teratomas and subsequently destroy the joint. Rheumatology (Oxford) 2003, 42, 162-165.

[9] da Silva Meirelles L, Caplan AI, Nardi NB: In search of the in vivo identity of mesenchymal stem cells. Stem Cells 2008, 26, 2287-2299.

[10] Takahashi K, Tanabe K, Ohnuki M, Narita M, Ichisaka T, Tomoda K, Yamanaka S: Induction of pluripotent stem cells from adult human fibroblasts by defined factors. Cell 2007, 131, 861-872.

[11] Obokata H, Sasai Y, Niwa H, Kadota M, Andrabi M, Takata N, Tokoro M, Terashita Y, Yonemura S, Vacanti CA, Wakayama T: Bidirectional developmental potential in reprogrammed cells with acquired pluripotency. Nature 2014, 505, 676-680.

[12] Kern S, Eichler H, Stoeve J, Kluter H, Bieback K: Comparative analysis of mesenchymal stem cells from bone marrow, umbilical cord blood, or adipose tissue. Stem Cells 2006, 24, 1294-1301.

[13] Cai L, Johnstone BH, Cook TG, Tan J, Fishbein MC, Chen PS, March KL: IFATS collection: Human adipose tissue-derived stem cells induce angiogenesis and nerve sprouting following myocardial infarction, in conjunction with potent preservation of cardiac function. Stem Cells 2009, 27, 230-237.

[14] Torres FC, Rodrigues CJ, Stocchero IN, Ferreira MC: Stem cells from the fat tissue of rabbits: an easy-to-find experimental source. Aesthetic Plast Surg 2007, 31, 574-578.

[15] Tiryaki T, Findikli N, Tiryaki D: Staged stem cell-enriched tissue (SET) injections for soft tissue augmentation in hostile recipient areas: a preliminary report. Aesthetic Plast Surg 2011, 35, 965-971.

[16] Rehman J, Traktuev D, Li J, Merfeld-Clauss S, Temm-Grove CJ, Bovenkerk JE, Pell CL, Johnstone BH, Considine RV, March KL: Secretion of angiogenic and antiapoptotic factors by human adipose stromal cells. Circulation 2004, 16, 109, 1292-1298.

[17] Kucerova L, Kovacovicova M, Polak S, Bohac M, Fedeles J, Palencar D, Matuskova M: Interaction of human adipose tissue-derived mesenchymal stromal cells with breast cancer cells. Neoplasma 2011, 58, 361-370.

[18] Ma Z, Han D, Zhang P, Yang JF, Wang Y, Zhang Y, Yang D, Liu J: Utilizing Muscle-Derived Stem Cells to Enhance Long-Term Retention and Aesthetic Outcome of Autologous Fat Grafting: Pilot Study in Mice. Aesth Plast Surg 2012, 36, 186-192.

[19] Orbay H, Tobita M, Hyakusoku H, Mizuno H: Effects of adipose-derived stem cells on improving the viability of diced cartilage grafts. Plast Reconstr Surg 2012, 129, 369-377.

[20] Schultheiss J, Seebach C, Henrich D, Willhelm K, Barker JH, Frank J: Mesenchymal stem cell (MSC) and endothelial progenitor cell (EPC) growth and adhesion in six different bone graft substitutes. Eur J Trauma Emerg Surg 2011, 37, 635-644.

[21] Hao W, Dong J, Jiang M, Wu J, Cui F, Zhou D: Enhanced bone formation in large segmental radial defects by combining adipose-derived stem cells expressing bone morphogenetic protein 2 with nHA/RHLC/PLA scaffold. Int Orthop 2010, 34, 1341-1349.

[22] Stockmann P, Park J, von Wilmowsky C, Nkenke E, Felszeghy E, Dehner JF, Schmitt C, Tudor C, Schlegel KA: Guided bone regeneration in pig calvarial bone defects using autologous mesenchymal stem/progenitor cells-a comparison of different tissue sources. J Craniomaxillofac Surg 2012, 40, 310-320.

[23] Qi M, Hu J, Zou S, Zhou H, Han L: Mandibular distraction osteogenesis enhanced by bone marrow mesenchymal stem cells in rats. J Craniomaxillofac Surg 2006, 34, 283-289.

[24] Behnia H, Khojasteh A, Soleimani M, Tehranchi A, Atashi A: Repair of alveolar cleft defect with mesenchymal stem cells and platelet derived growth factors: a preliminary report. J Craniomaxillofac Surg 2012, 40, 2-7.

[25] Smith DM, Cooper GM, Afifi AM, Mooney MP, Cray J, Rubin JP, Marra KG, Losee JE: Regenerative surgery in cranioplasty revisited: the role of adipose-derived stem cells and BMP-2. Plast Reconstr Surg 2011, 128, 1053-1060.

[26] Glasberg SB, D’Amico R: Use of regenerative human acellular tissue (AlloDerm) to reconstruct the abdominal wall following pedicle TRAM flap breast reconstruction surgery. Plast Reconstr Surg 2006, 118, 8-15.

[27] Orbay H, Takami Y, Hyakusoku H, Mizuno H: Acellular Dermal Matrix Seeded with Adipose-Derived Stem Cells as a Subcutaneous Implant. Aesth Plast Surg 2011, 35, 756-763.

[28] Reichenberger MA, Mueller W, Schafer A: Adipose Derived Stem Cells Protect Skin Flaps Against IschemiaReperfusion Injury. Stem Cell Rev Rep 2012, 8, 854-862.

[29] Mesimäki K, Lindroos B, Törnwall J, Mauno J, Lindqvist C, Kontio R, Miettinen S, Suuronen R: Novel maxillary reconstruction with ectopic bone formation by GMP adipose stem cells. Int J Oral Maxillofac Surg 2009, 38, 201-209. 
[30] Warnke PH, Springer IN, Wiltfang J, Acil Y, Eufinger H, Wehmöller M, Russo PA, Bolte H, Sherry E, Behrens E, Terheyden H: Growth and transplantation of a custom vascularised bone graft in a man. Lancet 2004, 364, 766-770.

[31] Sándor GK, Tuovinen VJ, Wolff J, Patrikoski M, Jokinen J, Nieminen E, Mannerström B, Lappalainen OP, Seppänen R, Miettinen S: Adipose stem cell tissue-engineered construct used to treat large anterior mandibular defect: a case report and review of the clinical application of good manufacturing practice-level adipose stem cells for bone regeneration. J Oral Maxillofac Surg 2013, 71, 938-950.

[32] Hanson SE, Bentz ML, Hematti P: Mesenchymal stem cell therapy for nonhealing cutaneous wounds. Plast Reconstr Surg 2010, 125, 510-516.

[33] Wu Y, Chen L, Scott PG, Tredget EE: Mesenchymal stem cells enhance wound healing through differentiation and angiogenesis. Stem Cells 2007, 25, 2648-2659.

[34] Falanga V, Iwamoto S, Chartier M, Yufit T, Butmarc J, Kouttab N, Shrayer D, Carson P: Autologous bone marrow-derived cultured mesenchymal stem cells delivered in a fibrin spray accelerate healing in murine and human cutaneous wounds. Tissue Eng 2007, 13, 1299-1312.

[35] Yoshikawa T, Mitsuno H, Nonaka I, Sen Y, Kawanishi K, Inada Y, Takakura Y,Okuchi K, Nonomura A: Wound therapy by marrow mesenchymal cell transplantation. Plast Reconstr Surg 2008, 121, 860-877.

[36] Leonardi D, Oberdoerfer D, Fernandes MC, Meurer RT, Pereira-Filho GA, Cruz P, Vargas M, Chem RC, Camassola M, Nardi NB: Mesenchymal stem cells combined with an artificial dermal substitute improve repair in full-thickness skin wounds. Burns 2012, 38, 1143-1150.

[37] Öksüz S, Ülkür E, Öncül O, Köse GT, Küçükodaci Z, Urhan M: The effect of subcutaneous mesenchymal stem cell injection on statis zone and apoptosis in an experimental burn model. Plast Reconstr Surg 2013, 131, 463-471.

[38] Yoshimura K, Asano Y, Aoi N, Kurita M, Oshima Y, Sato K, Inoue K, Suga H, Eto H, Kato H, Harii K: Progenitor-enriched adipose tissue transplantation as rescue for breast implant complications. Breast J 2010, 16, 169-175.

[39] Tanikawa DY, Aguena M, Bueno DF, Passos-Bueno MR, Alonso N: Fat grafts supplemented with adipose-derived stromal cells in the rehabilitation of patients with craniofacial microsomia. Plast Reconstr Surg 2013, 132, 141-152.

[40] Pathomvanich D, Palakurthi R: Application of stem cells in hair restoration surgery. In: Hair restoration Surgery in Asians. Eds.: Pathomvanich D, Imagawa K, Springer, New York 2010, 279-284.

\section{Address for correspondence:}

Maciej Koźlik

Department of Plastic Surgery

Wroclaw Medical University

Jana Pawła II 2

57-320 Polanica-Zdrój

Poland

Tel.: +48 696026312

E-mail: maciej.kozlik@gmail.com

Conflict of interest: None declared

Received: 22.12 .2013

Revised: 20.02.2014

Accepted: 15.10.2014 